OPEN ACCESS

Edited by:

Seyed Javad Mowla,

Tarbiat Modares University, Iran

Reviewed by:

Ji Heon Noh,

Chungnam National University,

South Korea

Damjan Glavav̌,

University of Ljubljana, Slovenia

*Correspondence:

Yongbin Yu

vxi74bv@163.com

Hongyan $\mathrm{Ge}$

exb0zzj@163.com

Specialty section:

This article was submitted to

RNA

a section of the journal

Frontiers in Genetics

Received: 23 June 2020

Accepted: 27 October 2020

Published: 17 November 2020

Citation:

Chen X, Zhao S, Li Q, XU C, YU Y and $\mathrm{Ge} H$ (2020) LnCRNA NEAT1 Knockdown Inhibits Retinoblastoma Progression by miR-3619-5p/LASP1

Axis. Front. Genet. 11:574145.

doi: 10.3389/fgene.2020.574145

\section{LncRNA NEAT1 Knockdown Inhibits Retinoblastoma Progression by miR-3619-5p/LASP1 Axis}

\author{
Xuedong Chen ${ }^{1}$, Shiyong Zhao'2, Qingjun $\mathrm{Li}^{1}$, Caicai Xu', Yongbin $\mathrm{Yu}^{1 *}$ and \\ Hongyan $\mathrm{Ge}^{1 *}$ \\ ' Department of Ophthalmology, The First Affiliated Hospital of Harbin Medical University, Harbin, China, ${ }^{2}$ Department \\ of Hepatopancreatobiliary Surgery, The Second Affiliated Hospital of Harbin Medical University, Harbin, China
}

Retinoblastoma $(\mathrm{RB})$ is the most common intraocular tumor in childhood. Long noncoding RNA (IncRNA) nuclear paraspeckle assembly transcript 1 (NTAT1) has been reported to be related to RB progression. This study aims to study the molecular mechanism of NEAT1 in regulating cell cycle, proliferation, apoptosis, migration, and invasion in RB. The expression levels of NEAT1 and miR-3619-5p were detected by quantitative real-time polymerase chain reaction (qRT-PCR). The protein expression of LIM and SH3 domain protein 1 (LASP1) was measured by western blot. The proliferation of RB cells was analyzed by cell counting kit-8 (CCK-8) and cell colony formation assays. Cell migration and invasion were evaluated by transwell assay. Cell cycle and apoptosis were assessed by flow cytometry analysis. The association between miR-3619-5p and NEAT1 or LASP1 was predicted by starBase 3.0 database and identified by dualluciferase reporter assay. The effects of NEAT1 knockdown on the tumor growth in vivo were detected by in vivo tumor formation assay. NEAT1 expression was dramatically upregulated, and miR-3619-5p expression was obviously downregulated in RB tissues and cells compared with control groups. The protein level of LASP1 was obviously increased in RB tissues or cells relative to paracancerous normal tissues or cells, respectively. Functionally, NEAT1 silencing inhibited RB cell migration, invasion, and proliferation, whereas induced cell apoptosis and cell cycle arrest in RB; this phenomenon was partially abolished by miR-3619-5p inhibitor. Mechanistically, NEAT1 acted as a sponge of miR-3619-5p, and miR-3619-5p was associated with LASP1. In addition, NEAT1 knockdown decreased the volume and weight of RB tumor in vivo. Together, NEAT1 silencing repressed cell migration, invasion, and proliferation, whereas induced cell apoptosis and cycle arrest by sponging miR-3619-5p to inhibit LASP1 expression in RB cells. This study may provide a theoretical basis for RB therapy.

Keywords: retinoblastoma, IncRNAs, NEAT1, miR-3619-5p, LASP1

\section{INTRODUCTION}

Retinoblastoma (RB) is the most common intraocular tumor in childhood. It has been reported that 90\% RB patients are under 5 years old, and 20-30\% cases are neonates (Mehyar et al., 2020). The survival rate of RB at the early stage is about $100 \%$. However, advanced RB can lead to death owing to its metastases from brain to tissues (Villegas et al., 2013). Although much effort and progress have been done, the pathogenesis of RB progression is still fuzzy. 
Long non-coding RNAs (LncRNAs) are a class of transcripts > 200 nucleotides in length (Li et al., 2015). LncRNAs regulate gene expression at transcriptional and post-translational levels. LncRNA dysregulation is related to cancer progression, including RB (Li J. et al., 2016; Sheng et al., 2018) Nuclear paraspeckle assembly transcript 1 (NEAT1) also participates in RB progression. Wang et al. indicated that NEAT1 silencing repressed cell proliferation and promoted apoptosis by inhibiting miR-124 in RB (Wang L. et al., 2019). Zhong et al. (2019) investigated that NEAT1 knockdown suppressed cell metastasis, whereas promoted cell apoptosis by associating with miR-204 to inhibit CXC chemokine receptor 4 (CXCR4) expression in $\mathrm{RB}$. In this study, the regulatory mechanism of $\mathrm{RB}$ progression by NEAT1 was further explored.

MicroRNAs (miRNAs) are 18-22 nucleotides in length. And miRNAs work via regulating the $3^{\prime}$ untranslated region ( $3^{\prime}$ UTR) of target genes, which results in their mRNA degradation and the repression of protein translation (Hua et al., 2011). MiR-3619$5 \mathrm{p}$ is also associated with cancer development. Niu et al. (2015) suggested that miR-3619-5p inhibited lung cancer progression by modulating $\beta$-catenin $3^{\prime}$ UTR. Zhang et al. (2018) indicated that miR-3619-5p repressed cell metastasis by inhibiting betacatenin, cyclin-dependent kinase 2 (CDK2) and activating p21 in bladder carcinoma. In addition, CDKN1A-mediated miR-3619$5 \mathrm{p}$ was also reported to repress cell proliferation and promote cell apoptosis in prostate cancer (Li et al., 2017). However, the mechanism by which miR-3619-5p affects $\mathrm{RB}$ growth is still poorly understood.

LIM and SH3 domain protein 1 (LASP1) belongs to LIM family and plays a vital part in cell structure with acting as transmitting messages from cytoplasm to nucleus (Orth et al., 2015). LASP1 is indicated to be overexpressed in various cancers (Liu H. et al., 2019). Zheng et al. (2016) suggested that LASP1 silencing hindered cell proliferation and metastasis in esophageal cancer (Liu H. et al., 2019). Shimizu et al. (2013) investigated that LASP1 silencing induced cell cycle accumulation in G2 phase in oral cancer. In addition, LASP1 silencing inhibited cell migration in clear cell renal cell cancer (Yang et al., 2014). But there are few studies on the regulation of RB progression by LASP1.

In this study, NEAT1 expression was determined by quantitative real time polymerase chain reaction (qRT-PCR). Mechanistically, loss-of-function experiments were carried out to determine the impacts of NEAT1 on RB progression. Functionally, the association among NEAT1, miR-3619-5p and LASP1 was predicted by starBase 3.0 database and identified by dual-luciferase reporter assay. In addition, in vivo tumor formation assay was used to reveal the impacts of NEAT1 knockdown on $\mathrm{RB}$ progression in vivo.

\section{MATERIALS AND METHODS}

\section{Clinical Tissue Sample and Cell Culture}

Forty pairs of RB tissues and paracancerous normal tissues $(2 \mathrm{~cm}$ far from tumors) were collected during biopsy at The First Affiliated Hospital of Harbin Medical University and instantly placed in liquid nitrogen. The Ethics Committee of The First
Affiliated Hospital of Harbin Medical University allowed this study. RB patients signed the written informed consents.

BeNa Culture Collection (Beijing, China) supplied the human RB cell lines (Y-79 and SO-RB50) and human retinal epithelial cell ARPE-19. All cells were stored in liquid nitrogen and cultivated in RAPI-1640 medium (HyClone, Logan, UT, United States) containing $10 \%$ fetal bovine serum and $1 \%$ penicillin-streptomycin (HyClone) at $37^{\circ} \mathrm{C}$ in a humid condition with $5 \% \mathrm{CO}_{2}$.

\section{Cell Transfection}

Small interfering RNA targeting NEAT1 (si-NEAT1), the overexpression plasmid of NEAT1 (NEAT1), miR-3619-5p mimic, miR-3619-5p inhibitor, the overexpression vector of LASP1 (pc-LASP1), small hairpin RNA against NEAT1 (sh-NEAT1) and their control groups, siRNA negative control (si-NC), vector, miRNA NC, inhibitor NC, pc-NC and shNC, were purchased from GenePharma (Shanghai, China). si-NEAT1 and si-NC were used to determine the interfering efficiency of si-NEAT1. In order to verify the relationship among NEAT1, miR-3619-5p and LASP1, the si-NEAT1, miR-3619$5 \mathrm{p}$ mimic/inhibitor or pc-LASP1 was transfected into cells with control groups. Cell transfection was carried out using Lipofectamine 2000 reagent (Thermo Fisher, Waltham, MA, USA) according to the manufacturer's instructions. The primer sequences were si-NEAT1 5'-GGAGGAGTCAGGAGGAATA-3'; si-NC 5'-GGATGAGACGAGAGGGATA-3'; miR-3619-5p mimic 5 '-UCAGCAGGCAGGCUGGUGCAGC-3'; miRNA NC: $\quad 5^{\prime}$-UUUGUACUACACAAAAGUACUG-3'; miR-3619$5 \mathrm{p}$ inhibitor $5^{\prime}$-GCUGCACCAGCCUGCCUGCUGA-3' and inhibitor NC 5' -CAGUACUUUUGUGUAGUACAAA-3'.

\section{RNA Extraction and qRT-PCR}

RB tissues and cells were lysed using RNAiso Plus (TaKaRa, Dalian, China). Then, RNA was extracted with RNAprep Pure Tissue kit (Tiangen, Beijing, China). The cDNA was amplified by a reagent kit (TaKaRa). A SYBR ${ }^{\circledR}$ Premix DimerEraser Kit (TaKaRa) was utilized to detect the expression levels of NEAT1, miR-3619-5p and LASP1. U6 snRNA and GAPDH were utilized to normalize the miRNA and lncRNA/mRNA. The sense and anti-sense primers were: NEAT1 5'-CTTCCTCCCTTTAACTTATCCATTCAC-3' and 5'-CTCTTCСТCCACCATTACCAACAATAC-3'; miR-3619-5p $5^{\prime}$-TCATCAGCAGGCAGGCTGGTGC- $3^{\prime}$ and $5^{\prime}$-GTGCAGGG TCCGAGGT-3' ${ }^{\prime}$ LASP $15^{\prime}$-CTGTCTCTGCCTTATAGCAACAC$3^{\prime}$ and $5^{\prime}$-CATCTCGAA CCTGGCTGTTTG-3'; U6 5'-TGCG GGTGCTCGCTTCGGCAGC- $3^{\prime}$ and $5^{\prime}$-GTGCAGGGTCCGAG GT-3'; GAPDH 5'-TATGATGATATCAAGAGGGTAGT-3' and 5'-TGTATCCAAACTCATTGTCATAC-3'.

\section{CCK-8 Assay}

Y-79 and SO-RB50 cells were cultivated in 96-well plate for $24 \mathrm{~h}$. Then, $10 \mu \mathrm{L}$ CCK-8 was added into plate after cell transfection. Cells were continued to culture for $4 \mathrm{~h}$ and results were analyzed by measuring the absorbance at $450 \mathrm{~nm}$ with microscope reader. 


\section{Transwell Assay}

The invasion and migration abilities of Y-79 and SO-RB50 cells were determined by transwell chamber with or without Matrigel (Corning, Shanghai, China), respectively. Briefly, cells were suspended in medium without serum. Following cells were added in the upper chamber. Medium with 10\% FBS was added into down chamber. Then cells were cultivated for $24 \mathrm{~h}$, following supernatant was discarded. Cells were incubated with methanol for $30 \mathrm{~min}$ and crystal violet for $20 \mathrm{~min}$. Results were visualized by microscope at a $100 \times$ magnification.

\section{Cell Colony Formation Assay}

Cells were cultivated in 6-well plate (500 cells per well) for 2 weeks. The proliferating colonies were stained with $1 \%$ crystal violet. Then colonies numbers were calculated and results were analyzed. A colony was defined when its cell number more than 50 .

\section{Flow Cytometry Assay}

Cell cycle and apoptosis were analyzed by cell cycle and apoptosis detection kit (Beyotime, Shanghai, China). In short, Y-79 and SORB50 cells were harvested after digested with trypsin (Thermo Fisher Scientific), and cells were washed with cold phosphate buffered solution (PBS). Then, cells were suspended in PBS, following were centrifuged. Cells were subsequently fixed with $70 \%$ anhydrous ethanol for $30 \mathrm{~min}$. Cells were centrifuged and supernatant was removed. Dye buffer, propidium iodide (PI) and RNase A were added and were incubated with cells at $37^{\circ} \mathrm{C}$ for $30 \mathrm{~min}$. Samples were assessed by flow cytometry (BD Biosciences, San Diego, CA, United States).

\section{Dual-Luciferase Reporter Assay}

StarBase 3.0 online database was employed to investigate the association between miR-3619-5p and NEAT1 or LASP1. The wide-type (WT) NEAT1 containing the binding sites of miR3619-5p and mutant (MUT) NEAT1 were amplified and inserted into the pGL3 vector (Promega Corporation, Fitchburg, WI, United States), named as WT-NEAT1 and MUT-NEAT1. The WT-LASP1-3'UTR and MUT-LASP1-3'UTR were synthesized and inserted into the pGL3 vector, named as WT-LASP1-3'UTR and MUT-LASP1-3'UTR. Then, the plasmids were transfected into cells with miR-3619-5p mimic or miRNA NC using Lipofectamine 2000 reagent according to the manufacturer's instructions. The results were detected by dual-luciferase reporter system. Renilla luciferase activity was chosen as a reference.

\section{Western Blot}

Samples were treated with RIPA buffer (Thermo Fisher Scientific). Then the lysate was loaded on 10\% SDS-PAGE. Then bands were transferred onto polyvinylidene fluoride membrane. Bands were blocked in the 5\% not-fat milk. The membranes were incubated with primary antibodies. Subsequently, the membranes were washed and incubated with secondary antibody labeled with horseradish peroxidase. Protein bands were visualized by ECL system (Pierce, Rockford, IL, United States). GAPDH was used as a reference. The primary antibodies were anti-LASP1 (1:3000; Proteintech, Rosemount, MN, United States) and anti-GAPDH (1:4000; Proteintech).

\section{RNA Pull-Down Assay}

Y-79 and SO-RB50 cells were grown for $16 \mathrm{~h}$, si-NEAT1 and si-NC were severally transfected into cells according to the manufacturer's instructions. $36 \mathrm{~h}$ later, biotinylated mi-3619-5p was transfected into Y-79 and SO-RB50 cells and cells were continued to culture $48 \mathrm{~h}$. Following cells were collected and lysed using lysis buffer. Then lysates were incubated with Streptavidincoupled Dynabeads (Invitrogen, Carlsbad, CA, United States) and proteinase K (Millipore, Billerica, MA, United States), respectively. The amount of ASAP1 was detected by qRT-PCR.

\section{In vivo Tumor Formation Assay}

RB Cells $\left(5 \times 10^{6}\right)$ transfected with sh-NEAT1 or sh-NC were injected into six-week old nude mice. Then, mice were continued to feed for 7 days. The tumor volume was recorded every 7 days. All nude mice were sacrificed at 28th day after injection. The tumors were excised and weight was analyzed. This study was approved by Animal Care and Use Committee of The First Affiliated Hospital of Harbin Medical University.

\section{Statistical Analysis}

Data analysis was carried out based on $\geq 3$ independent experiments. All data were assessed by GraphPad Prism 5.0 (GraphPad Software, La Jolla, CA, United States) or mage J software (NIH, Bethesda, MD, United States). Data was represented as mean $\pm \mathrm{SD}$. The comparison in study was evaluated by Student's $t$-test, one-way analysis of variance or chi-square test. $P<0.05$ was considered statistically significant.

\section{RESULTS}

\section{NEAT1 Expression Level Was Dramatically Upregulated in Retinoblastoma Tissues and Cells}

In order to determine the expression characteristics of NEAT1 in RB tissues and cells, its expression was detected by qRT-PCR. Results showed that expression level of NEAT1 was obviously upregulated in RB tissues compared with paracancerous normal tissues (Figure 1A). Additionally, qRT-PCR analysis revealed that the expression level of NEAT1 was higher in both Y-79 and SORB50 cells than in ARPE-19 (Figure 1B). These data investigated that NEAT1 was overexpressed in RB tissues and cells.

\section{NEAT1 Regulated Cell Migration, Invasion, Proliferation, Cycle Arrest, and Apoptosis in RB}

To explore the functional effects of NEAT1 on RB cell progression, the silencing efficiency of si-NEAT1 was firstly evaluated by qRT-PCR. Results showed that NEAT1 was dramatically repressed after si-NEAT1 transfection in Y-79 and SO-RB50 cells (Figure 2A). Then, the impacts of NEAT1 knockdown on RB progression were determined. CCK- 8 assay 
A

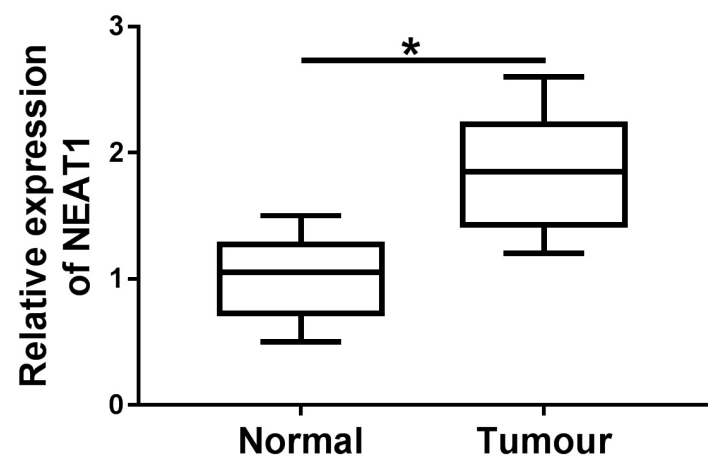

B

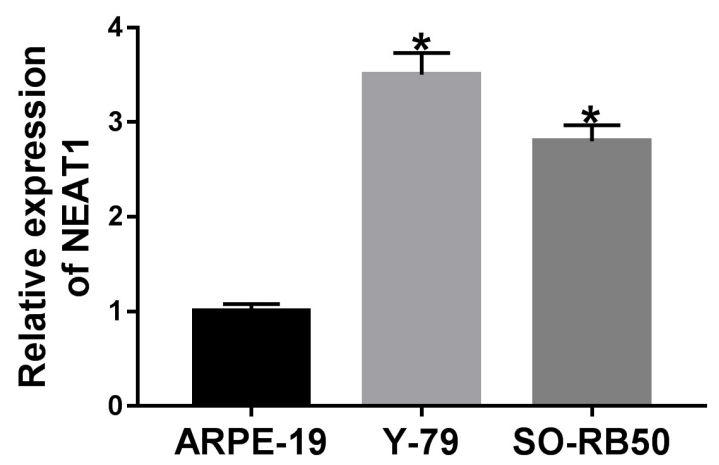

FIGURE 1 | NEAT1 expression level was dramatically up-regulated in RB tissues and cells. (A) The expression of NEAT1 in RB tissues and adjacent normal tissues was detected by qRT-PCR. (B) NEAT1 expression level in RB cells and ARPE-19 cells was assessed by qRT-PCR. ${ }^{*} P<0.05$.

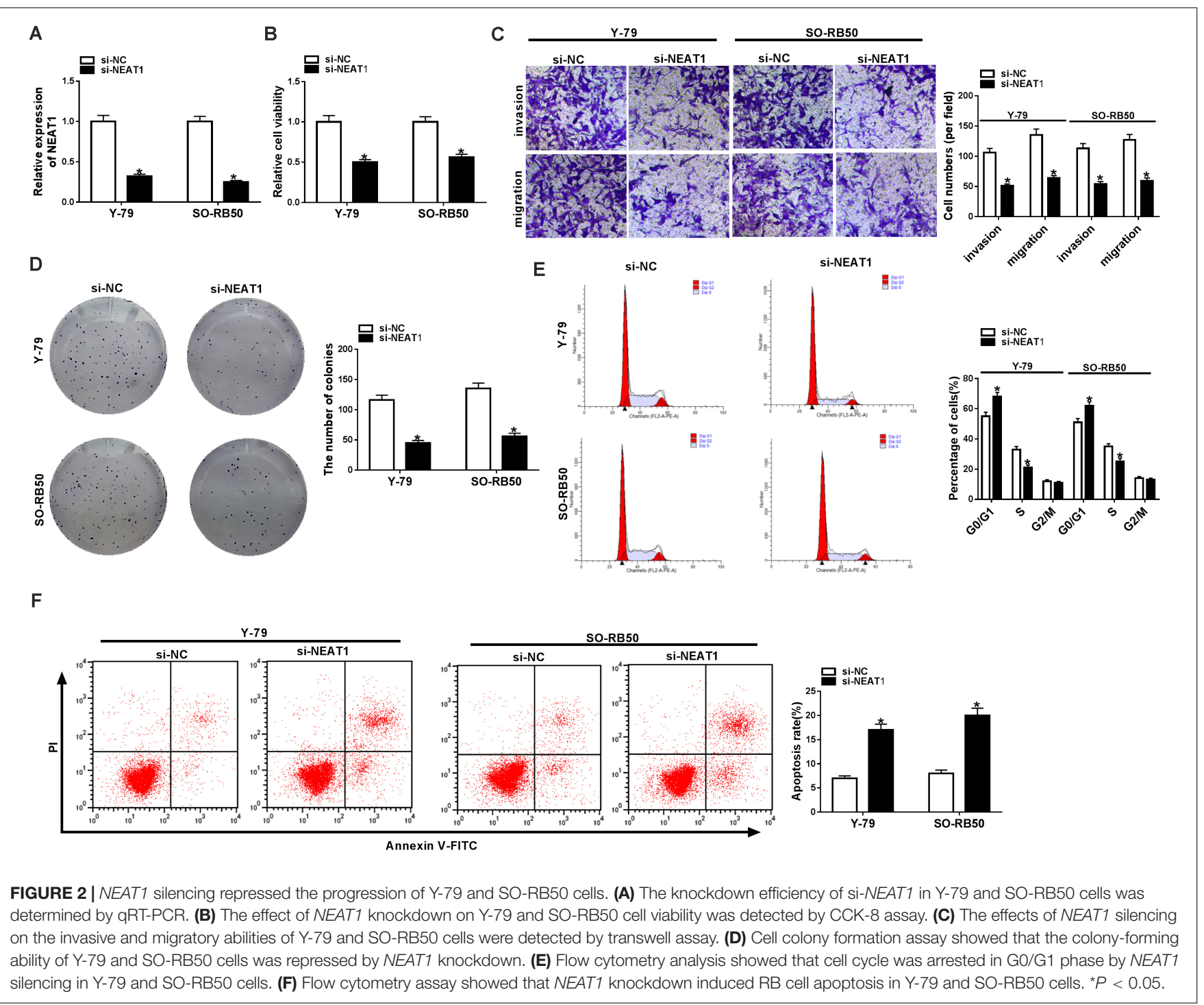




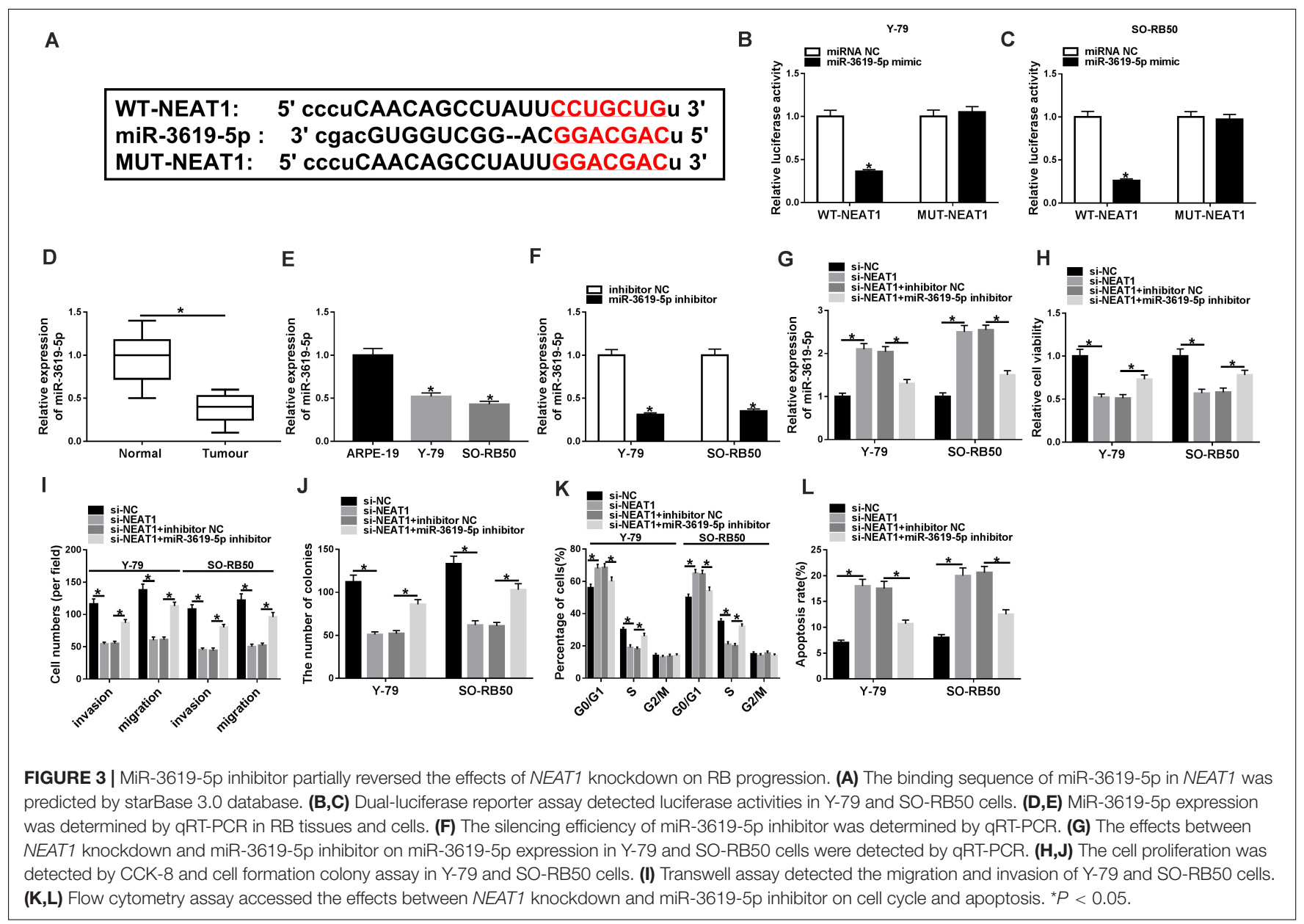

explained that the viability of Y-79 and SO-RB50 cells was inhibited by NEAT1 knockdown (Figure 2B). Transwell assay demonstrated that NEAT1 silencing inhibited the invasive and migratory abilities of Y-79 and SO-RB50 cells (Figure 2C). Cell colony formation assay showed that cell colony-forming ability was repressed by NEAT1 downregulation in Y-79 and SO-RB50 cells (Figure 2D). In addition, flow cytometry analysis revealed that NEAT1 knockdown induced cell cycle arrest in G0/G1 phase and apoptosis in Y-79 and SO-RB50 cells (Figures 2E,F). In order to further demonstrate that NEAT1 acted as an oncogene in RB progression, the effects of NEAT1 overexpression on the viability and apoptosis of normal retina cells (ARPE-19) were revealed. Results showed that ectopic NEAT1 expression had no impact on cell viability and apoptosis (Supplementary Figures 1A,B). All data suggested that NEAT1 contributed to RB development.

\section{NEAT1 Knockdown Repressed RB Cell Progression by Sponging miR-3619-5p}

To understand the molecular mechanism of NEAT1 knockdown in regulating $\mathrm{RB}$ cell development, the associated gene with NEAT1 was predicted by starBase 3.0 database. Figure $3 \mathrm{~A}$ showed that NEAT1 contained the binding sites of miR-3619-5p. Further, dual-luciferase reporter assay showed that the luciferase activity of WT-NEAT1 + miR-3619-5p group was greatly inhibited in Y-79 and SO-RB50 cells, whereas the luciferase activity of MUT-NEAT1 + miR-3619-5p group had no significant change (Figures 3B,C). Subsequently, miR-3619-5p expression was detected by qRT-PCR in RB tissues and cells. Results showed that miR-3619-5p was downregulated in RB tissues and cells relative to control groups (Figures 3D,E). These outcomes elucidated that NEAT1 was a sponge of miR-3619-5p.

In order to detect the effects between NEAT1 knockdown and miR-3619-5p inhibitor on RB progression, the silencing efficiency of miR-3619-5p inhibitor was firstly determined by qRT-PCR. Results revealed that miR-3619-5p inhibitor obviously repressed the expression of miR-3619-5p (Figure 3F). Subsequently, qRT-PCR analysis suggested that NEAT1 silencing up-regulated miR-3619-5p expression, whereas miR-3619-5p inhibitor partially reversed this effect (Figure 3G). CCK-8 and cell colony formation assays showed that NEAT1 repression inhibited RB cell proliferation, whereas this phenomenon was partially abolished by miR-3619-5p inhibitor (Figures $\mathbf{3 H}, \mathbf{J}$ ). Transwell assay showed that cell invasive and migratory abilities were obviously repressed by NEAT1 knockdown in Y-79 and SO-RB50 cells; however, the inhibition effect was decreased by miR-3619-5p inhibitor (Figure 3I). Further, flow cytometry analysis showed that NEAT1 knockdown induced the cycle arrest 


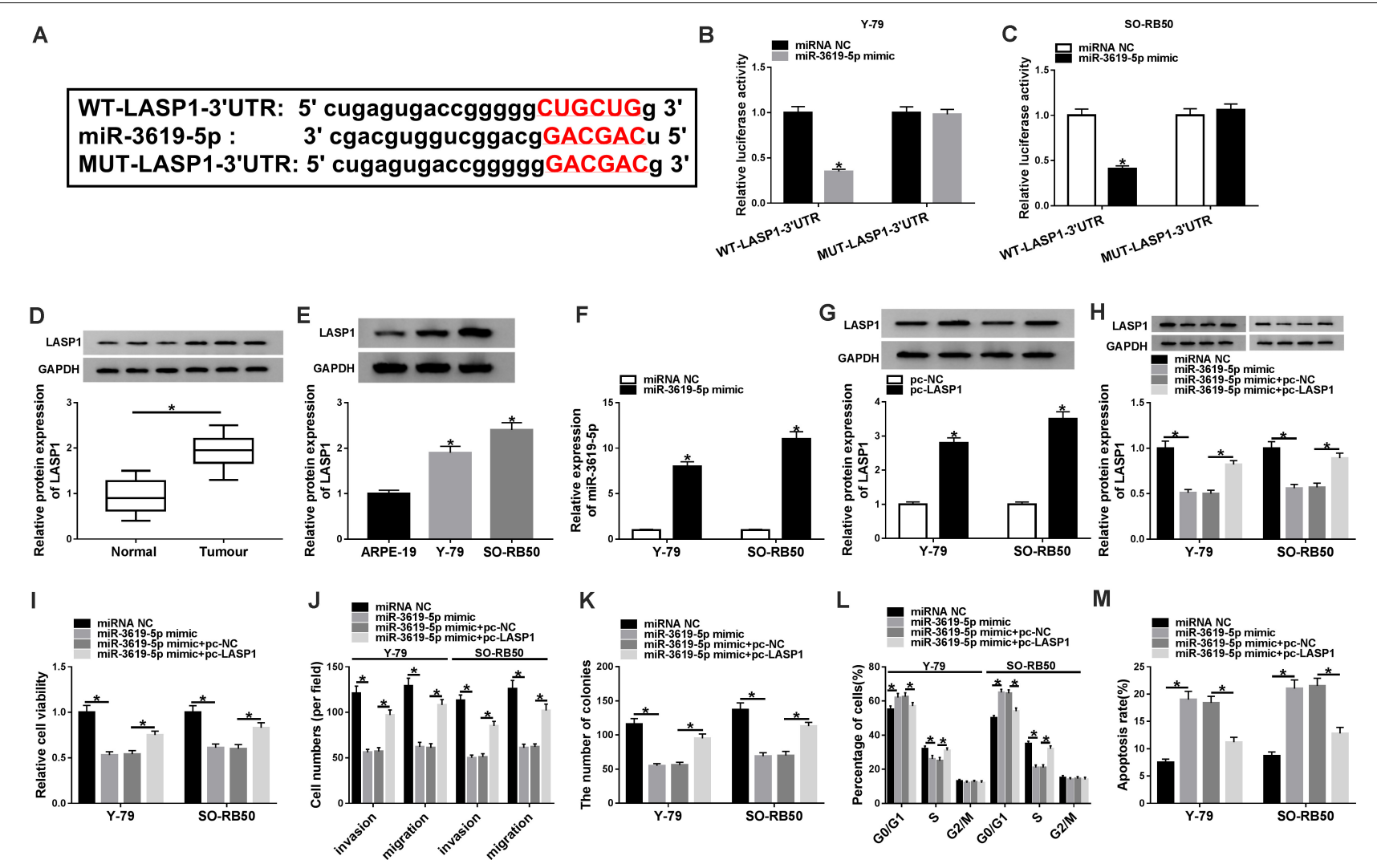

FIGURE 4 | MiR-3619-5p repressed RB cell progression by sponging LASP1. (A) The starBase 3.0 database predicted the binding sites between miR-3619-5p and LASP1. (B,C) The luciferase activities were detected by dual-luciferase reporter assay. (D,E) The protein level of LASP1 was determined by western blot in RB tissue and cells. (F) The transfection efficiency of miR-3619-5p mimic was detected by qRT-PCR. (G) Western blot detected the transfection efficiency of pc-LASP1 in Y-79 and SO-RB50 cells. (H) The impacts between miR-3619-5p mimic and LASP1 overexpression on LASP1 expression were determined by western blot in Y-79 and SO-RB50 cells. (I,K) The cell proliferation was detected by CCK-8 and cell colony formation assays in Y-79 and SO-RB50 cells. (J) The invasive and migratory abilities of RB cells were determined by transwell assay in Y-79 and SO-RB50 cells. (L,M) Flow cytometry analysis revealed that the effects between miR-3619-5p mimic and LASP1 overexpression on the cell cycle and apoptosis of Y-79 and SO-RB50 cells. ${ }^{*} P<0.05$.

and apoptosis of Y-79 and SO-RB50 cells, and miR-3619-5p inhibitor partly restored these effects (Figures 3K,L). These data indicated that NEAT1 silencing regulated RB progression by binding to miR-3619-5p.

\section{LASP1 Overexpression Partially Attenuated the Effects of miR-3619-5p Mimic on the Cell Proliferation, Migration, Invasion, Cell Cycle and Apoptosis in RB}

In order to further determine that how miR-3619-5p regulated $\mathrm{RB}$ development, the targeting gene of miR-3619-5p was predicted. Results revealed that LASP1-3'UTR contained the binding sites of miR-3619-5p (Figure 4A). Subsequently, dualluciferase reporter assay investigated that the luciferase activity of WT-LASP1-3'UTR + miR-3619-5p group was significantly decreased in both Y-79 and SO-RB50 cells; however, the luciferase activity of MUT-LASP1-3'UTR + miR-3619-5p group was not changed (Figures $4 \mathrm{~B}, \mathrm{C}$ ). In addition, LASP1 protein expression was detected in RB tissues and cells, and western blot analysis showed that the protein level of LASP1 was significantly upregulated in $\mathrm{RB}$ tissues or cells compared with normal tissues or cells, respectively (Figures $4 \mathrm{D}, \mathrm{E}$ ). These results suggested that miR-3619-5p targeted LASP1.

To investigate the effects between miR-3619-5p mimics and LASP1 overexpression on $\mathrm{RB}$ progression, the transfection efficiency of miR-3619-5p mimic and pc-LASP1 was firstly detected. The qRT-PCR analysis showed that miR-3619-5p mimic strongly increased the expression of miR-3619-5p (Figure 4F), and western blot assay explained that LASP1 protein level was apparently up-regulated after pc-LASP1 transfection (Figure 4G). Then western blot analysis explained that miR3619-5p mimic inhibited LASP1 expression and this effect was decreased by LASP1 overexpression (Figure $4 \mathbf{H}$ ). Based on the above data, CCK-8 and cell colony formation assay showed that miR-3619-5p mimic inhibited the viability and colony-forming ability of Y-79 and SO-RB50 cells, whereas LASP1 overexpression partly attenuated this inhibition effect (Figures 4I,K). Similarly, the cell invasive and migratory abilities of $\mathrm{RB}$ were repressed by miR-3619-5p mimic; however, this phenomenon was partially abolished by LASP1 overexpression (Figure 4J). In addition, 
A

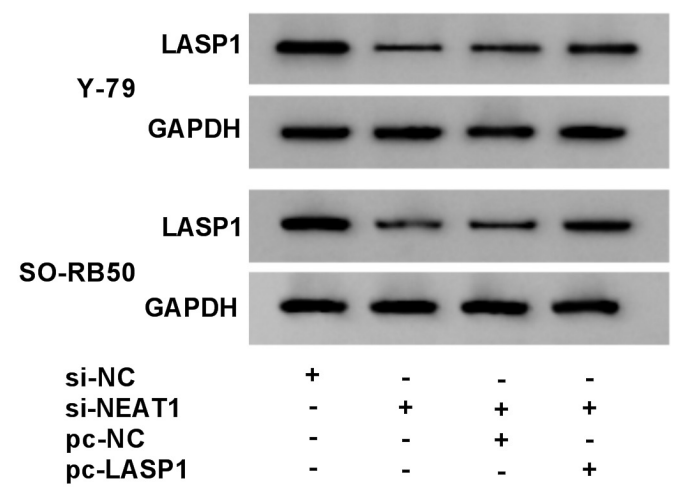

B

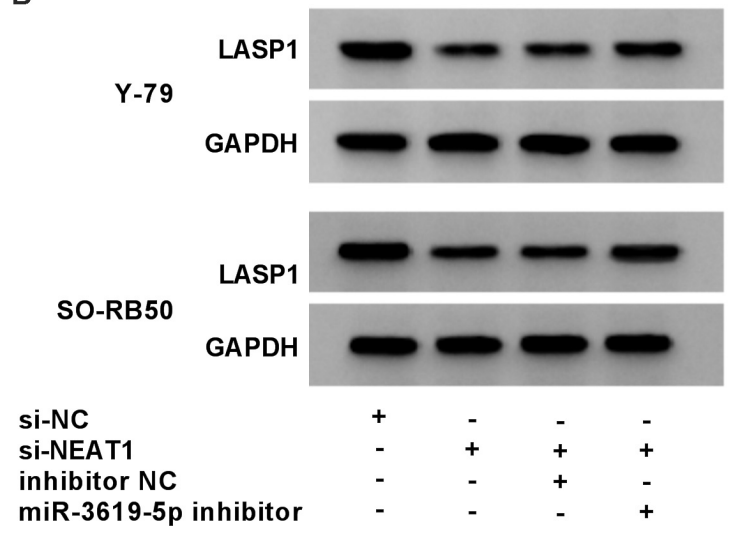

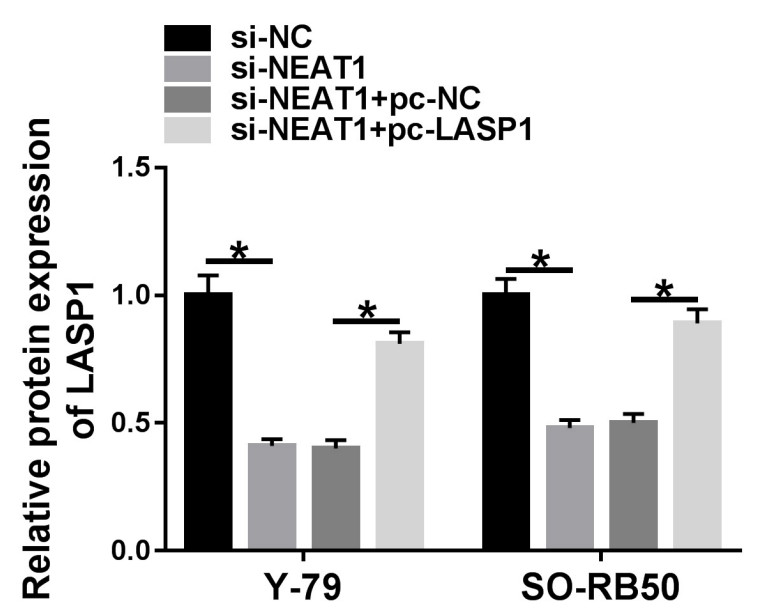

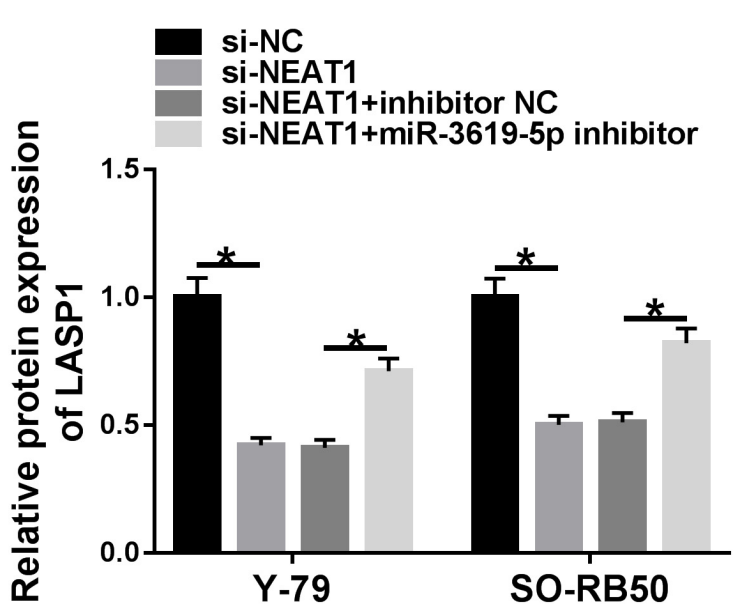

FIGURE 5 | NEAT1 silencing repressed the protein level of LASP1 by regulating miR-3619-5p. (A) The effects between NEAT1 knockdown and LASP1 overexpression on LASP1 protein expression were revealed by western blot. (B) Western blot demonstrated that NEAT1 knockdown repressed the protein expression of LASP1, which was decreased by miR-3619-5p inhibitor. ${ }^{\star} P<0.05$.

miR-3619-5p mimic induced cell cycle arrest in G0/G1 phase and apoptosis in Y-79 and SO-RB50 cells, but these impacts were partly restored by pc-LASP1 (Figures 4L,M). Collectively, these results demonstrated that miR-3619-5p inhibited RB cell progression by targeting LASP1-3'UTR.

\section{NEAT1 Knockdown Downregulated the Protein Expression of LASP1 by Sponging miR-3619-5p}

To further determine the relationship among NEAT1, miR3619-5p and LASP1, the effects between NEAT1 knockdown and LASP1 overexpression or miR-3619-5p inhibitor on LASP1 protein expression were detected in Y-79 and SO-RB50 cells. Western blot revealed that NEAT1 silencing repressed LASP1 protein expression, whereas this effect was partially abolished by LASP1 overexpression or miR-3619-5p inhibitor (Figures 5A,B). In order to further illustrate whether NEAT1 affected LASP1 expression by sponging miR-3619-5p, RNA pull-down assay was employed. Results showed that the enrichment of LASP1 by miR-3619-5p was dramatically increased after NEAT1 silencing (Supplementary Figure 2). Thus, all data suggested that NEAT1 up-regulated LASP1 expression by sponging miR-3619-5p.

\section{NEAT1 Knockdown Repressed Rb Growth in vivo}

The effects of NEAT1 silencing on RB growth in vivo were explored. Results showed that tumor volume and weight were dramatically decreased by NEAT1 silencing (Figures 6A,B). Further, the effects of NEAT1 knockdown on miR-3619-5p expression and LASP1 protein level were analyzed in vivo. QRT-PCR revealed that the interfering vector of NEAT1 was successfully built (Figure 6C) and NEAT1 knockdown upregulated miR-3619-5p expression (Figure 6D). Western blot analysis showed NEAT1 silencing inhibited LASP1 protein level 


\section{A}

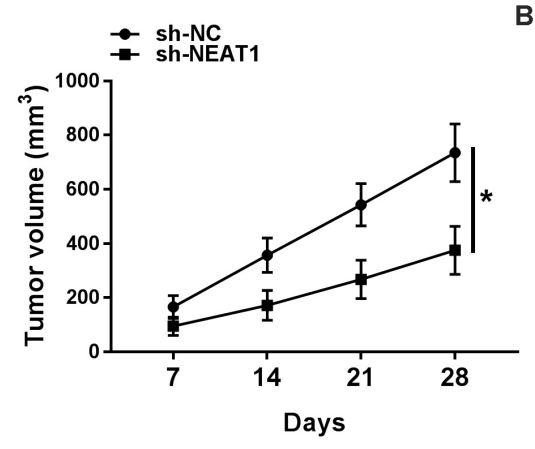

B

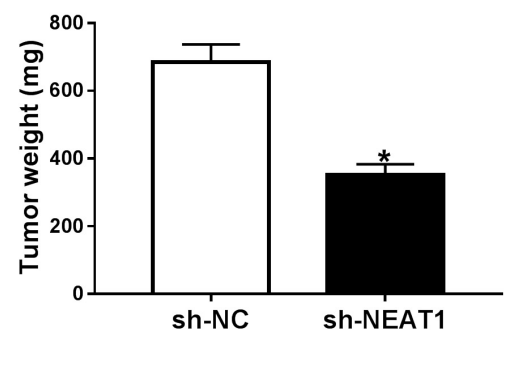

C

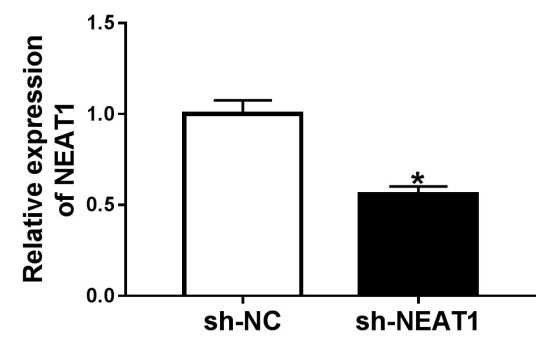

D

E
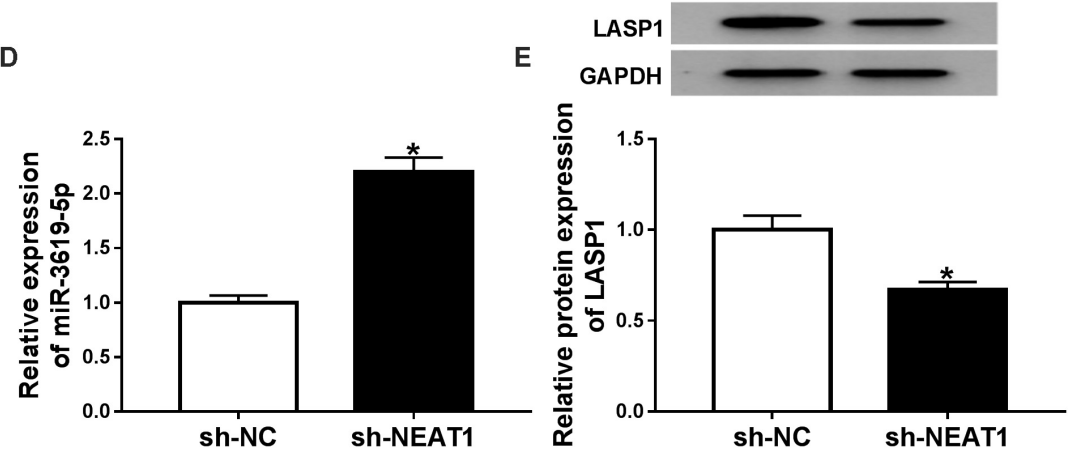

sh-NC

FIGURE 6 | NEAT1 knockdown inhibited RB growth in vivo. (A) Tumor volume was inhibited by NEAT1 knockdown. (B) The weight of tumors was reduced after NEAT1 silencing in vivo. (C) The interfering efficiency of sh-NEAT1 was determined by qRT-PCR. (D) QRT-PCR showed that NEAT1 knockdown up-regulated miR-3619-5p expression in vivo. (E) Western blot analysis showed that NEAT1 knockdown repressed the protein level of LASP1 in vivo. ${ }^{\star} P<0.05$.

(Figure 6E). From these data, it was concluded that NEAT1 knockdown inhibited RB growth by regulating miR-3619-5p and LASP1 in vivo.

\section{DISCUSSION}

LncRNAs were revealed to work by sponging miRNAs to repress mRNA expression (Dong et al., 2018). LncRNAs can be employed to as biomarkers for cancer treatment based on LncRNAs regulating cell development. LncRNA NEAT1, a scaffold factor, has been investigated to take part in diverse cancer progression.

Zhuang et al. (2020) indicated NEAT1 was up-regulated in colon cancer and its overexpression promoted the metastasis of colon cancer cells. NEAT1 was indicated to be overexpressed in pancreatic cancer and increased cell proliferation and metastases in pancreatic cancer cells (Feng et al., 2020). Pang et al. (2019) investigated that NEAT1 expression was increased and NEAT1 elevated cell cycle progression in breast cancer. In addition, $\mathrm{Yu}$ et al. (2019) suggested that NEAT1 knockdown promoted cell apoptosis in lung cancer . Similarly, we found NEAT1 expression was up-regulated and NEAT1 knockdown inhibited cell proliferation, migration and invasion, and promoted cell cycle arrest and apoptosis in RB. Meanwhile, our data showed that NEAT1 overexpression enhanced cell viability, whereas had no effect on cell apoptosis in normal retina cells (ARPE-19). These results indicated that NEAT1 functioned as an oncogene in $\mathrm{RB}$ progression.
MiR-3619-5p was indicated to repress the proliferation and metastasis of RB cells (Yan et al., 2019). In addition, miR3619-5p promoted cell apoptosis in papillary thyroid carcinoma (Wang G. et al., 2019). Li et al. (2017) explained that miR$3619-5 \mathrm{p}$ promoted cell cycle arrest in prostate cancer cells. Coincidentally, our study showed that NEAT1 was a sponge of miR-3619-5p. MiR-3619-5p was revealed that its overexpression repressed cell proliferation, migration and invasion, and promoted cell cycle arrest and apoptosis in RB. These data were consistent with previous results. Besides, miR-3619-5p expression was downregulated in RB tissues and cells. Collectively, all data suggested that NEAT1 promoted RB development by sponging miR-3619-5p.

Further, we found miR-3619-5p targeted LASP1 and inhibited LASP1 expression, and LASP1 could decrease the inhibition effects of miR-3619-5p on RB progression. All the above results suggested that LASP1 had promotion effects on RB progression. Liu H. et al. (2019) indicated that the inhibition effect of miR-143 on cell metastasis was impaired by LASP1 in human esophageal cancer. In esophageal cancer, circ-0004370 silencing suppressed cell proliferation and induced cell apoptosis, whereas these effects were decreased by LASP1 (Zhang et al., 2019). Our results were similar to these findings. In addition, our studies suggested that LASP1 inhibited cell cycle accumulation in G0/G1 phase. Nevertheless, LASP1 was found to induce cell cycle arrest in G2/M phase in thyroid cancer and gallbladder cancer ( $\mathrm{Li} \mathrm{Z}$. et al., 2016; Liu W. et al., 2019). The reason for this phenomenon maybe was that the regulatory mechanism of LASP1 in cell cycle was 
discrepant in various cancers. The finding in this part investigated that miR-3619-5p inhibited RB progression by targeting LASP1.

Taken together, NEAT1 and LASP1 expression were dramatically up-regulated, and miR-3619-5p expression was obviously downregulated in RB tissues and cells. NEAT1 knockdown inhibited cell proliferation and metastasis, whereas promoted cell apoptosis and cell cycle arrest in RB. Enforced NEAT1 expression fas had no effect on cell viability and apoptosis in normal retina cells. MiR-3619-5p inhibitor decreased the inhibition effects of NEAT1 knockdown on RB progression. NEAT1 was a sponge of miR-3619-5p and miR-3619-5p was associated with LASP1. Besides, NEAT1 knockdown repressed $\mathrm{RB}$ growth in vivo. Therefore, we came into a conclusion that NEAT1 silencing inhibited RB cell progression by downregulating LASP1 expression through sponging miR-3619-5p as showed in Supplementary Figure 3, which established a foundation for further study of RB progression and provided a theory evidence in studying RB therapy.

\section{DATA AVAILABILITY STATEMENT}

The original contributions presented in the study are included in the article/Supplementary Material, further inquiries can be directed to the corresponding author/s.

\section{ETHICS STATEMENT}

The studies involving human participants were reviewed and approved by The First Affiliated Hospital of Harbin Medical University. The patients/participants provided their written informed consent to participate in this study. The animal study was reviewed and approved by The First Affiliated Hospital of Harbin Medical University.

\section{REFERENCES}

Dong, P., Xiong, Y., Yue, J., Hanley, S. J. B., Kobayashi, N., Todo, Y., et al. (2018). Long non-coding RNA NEAT1: a novel target for diagnosis and therapy in human tumors. Front. Genet. 9:471. doi: 10.3389/fgene.2018.00471

Feng, Y., Gao, L., Cui, G., and Cao, Y. (2020). LncRNA NEAT1 facilitates pancreatic cancer growth and metastasis through stabilizing ELF3 mRNA. Am. J. Cancer Res. 10, 237-248.

Hua, Y., Duan, S., Murmann, A. E., Larsen, N., Kjems, J., Lund, A. H., et al. (2011). miRConnect: identifying effector genes of miRNAs and miRNA families in cancer cells. PLoS One 6:e26521. doi: 10.1371/journal.pone.0026521

Li, J., Zhuang, C., Liu, Y., Chen, M., Chen, Y., Chen, Z., et al. (2016). Synthetic tetracycline-controllable shRNA targeting long non-coding RNA HOXD-AS1 inhibits the progression of bladder cancer. J. Exp. Clin. Cancer Res. 35:99. doi: 10.1186/s13046-016-0372-5

Li, P., Chen, S., Chen, H., Mo, X., Li, T., Shao, Y., et al. (2015). Using circular RNA as a novel type of biomarker in the screening of gastric cancer. Clin. Chim. Acta 444, 132-136. doi: 10.1016/j.cca.2015.02.018

Li, S., Wang, C., Yu, X., Wu, H., Hu, J., Wang, S., et al. (2017). miR-3619-5p inhibits prostate cancer cell growth by activating CDKN1A expression. Oncol. Rep. 37, 241-248. doi: 10.3892/or.2016.5250

Li, Z., Chen, Y., Wang, X., Zhang, H., Zhang, Y., Gao, Y., et al. (2016). LASP1 induces proliferation, metastasis and cell cycle arrest at the G2/M phase

\section{AUTHOR CONTRIBUTIONS}

HG designed and supervised the study. XC conducted the experiments and drafted the manuscript. SZ conducted the experiments and supervised the study. YY collected and analyzed the data. QL contributed to the methodology and analyzed the data. CX edited the manuscript. All authors read and approved the final manuscript.

\section{FUNDING}

This study was supported by a grant from the Heilongjiang Postdoctoral Scientific Research Developmental Fund (No. LBH-Q17093).

\section{SUPPLEMENTARY MATERIAL}

The Supplementary Material for this article can be found online at: https://www.frontiersin.org/articles/10.3389/fgene. 2020.574145/full\#supplementary-material

Supplementary Figure 1 | NEAT1 overexpression had no effect on cell viability and apoptosis in ARPE-19 cells. (A) The influence of enforced NEAT1 expression on the viability of ARPE-19 cells was illustrated by CCK-8 assay. (B) The impact of NEAT1 overexpression on the apoptosis of ARPE-19 cells was demonstrated by flow cytometry assay. ns: not significantly.

Supplementary Figure 2 | NEAT1 silencing increased the enrichment of LASP1 by miR-3619-5p in Y-79 and SO-RB50 cells. (A) RNA pull-down assay was carried out to detect the amount of LASP1 enriched by miR-3619-5p in Y-79 and SO-RB50 cells.

Supplementary Figure 3 | The diagram of mechanism of NEAT1 in regulating $\mathrm{RB}$ process.

Supplementary Table 1 | Correlation between NEAT1 expression and clinical clinicopathological parameters of RB.

in gallbladder cancer by down-regulating S100P via the PI3K/AKT pathway. Cancer Lett. 372, 239-250. doi: 10.1016/j.canlet.2016.01.008

Liu, H., Zheng, M., Zhao, Y., and Zhang, S. (2019). miR-143 inhibits migration and invasion through regulating LASP1 in human esophageal cancer. Int. J. Clin. Exp. Pathol. 12, 466-476.

Liu, W., Wang, Z., Wang, C., and Ai, Z. (2019). Long non-coding RNA MIAT promotes papillary thyroid cancer progression through upregulating LASP1. Cancer Cell Int. 19, 194-194. doi: 10.1186/s12935-019-0913-z

Mehyar, M., Mosallam, M., Tbakhi, A., Saab, A., Sultan, I., Deebajah, R., et al. (2020). Impact of RB1 gene mutation type in retinoblastoma patients on clinical presentation and management outcome. Hematol. Oncol. Stem Cell Ther. 13, 152-159. doi: 10.1016/j.hemonc.2020.02.006

Niu, X., Liu, S., Jia, L., and Chen, J. (2015). Role of MiR-3619-5p in betacatenin-mediated non-small cell lung cancer growth and invasion. Cell. Physiol. Biochem. 37, 1527-1536. doi: 10.1159/000438520

Orth, M. F., Cazes, A., Butt, E., and Grunewald, T. G. P. (2015). An update on the LIM and SH3 domain protein 1 (LASP1): a versatile structural, signaling, and biomarker protein. Oncotarget 6, 26-42. doi: 10.18632/oncotarget.3083

Pang, Y., Wu, J., Li, X., Wang, C., Wang, M., Liu, J., et al. (2019). NEAT1/miR124/STAT3 feedback loop promotes breast cancer progression. Int. J. Oncol. 55, 745-754. doi: 10.3892/ijo.2019.4841

Sheng, L., Wu, J., Gong, X., Dong, D., and Sun, X. (2018). SP1-induced upregulation of lncRNA(PANDAR)predicts adverse phenotypes in 
retinoblastoma and regulates cell growth and apoptosis in vitro and in vivo. Gene 668, 140-145. doi: 10.1016/j.gene.2018.05.065

Shimizu, F., Shiiba, M., Ogawara, K., Kimura, R., Minakawa, Y., Baba, T., et al. (2013). Overexpression of LIM and SH3 Protein 1 leading to accelerated G2/M phase transition contributes to enhanced tumourigenesis in oral cancer. PLoS One 8:e83187. doi: 10.1371/journal.pone.0083187

Villegas, V. M., Hess, D. J., Wildner, A., Gold, A. S., and Murray, T. G. (2013). Retinoblastoma. Curr. Opin. Ophthalmol. 24, 581-588. doi: 10.1097/icu. 0000000000000002

Wang, G., Wang, X., and Jin, Y. (2019). LINC01410/miR-3619-5p/FOXM1 feedback loop regulates papillary thyroid carcinoma cell proliferation and apoptosis. Cancer Biother. Radiopharm. 34, 572-580. doi: 10.1089/cbr.2019. 2854

Wang, L., Yang, D., Tian, R., and Zhang, H. (2019). NEAT1 promotes retinoblastoma progression via modulating miR-124. J. Cell. Biochem. 120, 15585-15593. doi: $10.1002 /$ jcb. 28825

Yan, G., Su, Y., Ma, Z., Yu, L., and Chen, N. (2019). Long noncoding RNA LINC00202 promotes tumor progression by sponging miR-3619-5p in retinoblastoma. Cell Struct. Funct. 44, 51-60. doi: 10.1247/csf.18033

Yang, F., Zhou, X., Du, S., Zhao, Y., Ren, W., Deng, Q., et al. (2014). LIM and SH3 domain protein 1 (LASP-1) overexpression was associated with aggressive phenotype and poor prognosis in clear cell renal cell cancer. PLoS One 9:e100557. doi: 10.1371/journal.pone.0100557

Yu, P. F., Wang, Y., Lv, W., Kou, D., Hu, H. L., Guo, S. S., et al. (2019). LncRNA NEAT1/miR-1224/KLF3 contributes to cell proliferation, apoptosis and invasion in lung cancer. Eur. Rev. Med. Pharmacol. Sci. 23, 8403-8410. doi: 10.26355/eurrev_201910_19151
Zhang, Q., Miao, S., Han, X., Li, C., Zhang, M., Cui, K., et al. (2018). MicroRNA3619-5p suppresses bladder carcinoma progression by directly targeting betacatenin and CDK2 and activating p21. Cell Death Dis. 9:960. doi: 10.1038/ s41419-018-0986-y

Zhang, Z., Lin, W., Gao, L., Chen, K., Yang, C., Zhuang, L., et al. (2019). Hsa_circ_0004370 promotes esophageal cancer progression through miR1294/LASP1 pathway. Biosci. Rep. 39:BSR20182377. doi: 10.1042/BSR20182377

Zheng, J., Wang, F., Lu, S., and Wang, X. (2016). LASP-1, regulated by miR-203, promotes tumor proliferation and aggressiveness in human non-small cell lung cancer. Exp. Mol. Pathol. 100, 116-124. doi: 10.1016/j.yexmp.2015.11.031

Zhong, W., Yang, J., Li, M., Li, L., and Li, A. (2019). Long noncoding RNA NEAT1 promotes the growth of human retinoblastoma cells via regulation of miR-204/CXCR4 axis. J. Cell. Physiol. 234, 11567-11576. doi: 10.1002/jcp.27812

Zhuang, S. T., Cai, Y. J., Liu, H. P., Qin, Y., and Wen, J. F. (2020). LncRNA NEAT1/miR-185-5p/IGF2 axis regulates the invasion and migration of colon cancer. Mol. Genet. Genomic Med. 8:e1125. doi: 10.1002/mgg3.1125

Conflict of Interest: The authors declare that the research was conducted in the absence of any commercial or financial relationships that could be construed as a potential conflict of interest.

Copyright (c) 2020 Chen, Zhao, Li, Xu, Yu and Ge. This is an open-access article distributed under the terms of the Creative Commons Attribution License (CC BY). The use, distribution or reproduction in other forums is permitted, provided the original author(s) and the copyright owner(s) are credited and that the original publication in this journal is cited, in accordance with accepted academic practice. No use, distribution or reproduction is permitted which does not comply with these terms. 